\title{
Intrasylvian hematoma caused by ruptured middle cerebral artery aneurysms predicts recovery from poor-grade subarachnoid hemorrhage
}

\author{
Hitoshi Fukuda, MD, ${ }^{1}$ Kosuke Hayashi, MD, ${ }^{1}$ Takafumi Moriya, RT, ${ }^{2}$ Satoru Nakashita, MD,, ${ }^{2}$ \\ Benjamin W. Y. Lo, MD, ${ }^{3}$ and Sen Yamagata, MD'

\begin{abstract}
Departments of ${ }^{1}$ Neurosurgery and ${ }^{2}$ Radiology, Kurashiki Central Hospital, Kurashiki, Okayama, Japan; and ${ }^{3}$ Divisions of McMaster University, Hamilton, Ontario, Canada
\end{abstract} \\ Neurosurgery and Critical Care Medicine, Department of Clinical Epidemiology and Biostatistics, University of Toronto and
}

\begin{abstract}
OBJECT Intrasylvian hematoma (ISH) is a subtype of intracranial hematoma caused by aneurysmal rupture and often presents with a poor initial neurological grade; it is not well studied. The aim of this study was to elucidate outcomes of aneurysmal subarachnoid hemorrhage (SAH) with ISH.
\end{abstract}

METHODS Data for 97 patients with poor-grade SAH (World Federation of Neurosurgical Societies Grade IV or V) were retrospectively analyzed from a single-center, prospective, observational cohort database. Ultra-early surgical clipping, removal of hematoma, external decompression for brain swelling, and prevention of vasospasm by cisternal irrigation with milrinone were combined as an aggressive treatment. Characteristics and clinical courses of SAH with ISH were identified. The authors also evaluated any correlations between poor admission-grade SAH and ISH with good functional outcome.

RESULTS Patients with poor admission-grade SAH and with ISH were more likely to have initial cerebral edema $(p<$ 0.001, Mann-Whitney U-test), which significantly resolved over time ( $p<0.001$, Mann-Whitney U-test). These patients had a better chance of functional survival (modified Rankin Scale scores of 1-3; OR 5.75; 95\% Cl 1.36-24.3; $p=0.017$ ) at 6 months after hospital discharge, after adjustment for potential confounders such as younger age and better initial neurological grade by multivariable analysis.

CONCLUSIONS ISH predicted good functional recovery from poor-grade aneurysmal SAH.

http://thejns.org/doi/abs/10.3171/2014.10.JNS141658

KEY WORDS intracerebral hemorrhage; intrasylvian hematoma; middle cerebral artery aneurysm; subarachnoid hemorrhage; vasospasm; favorable outcome; vascular disorders

$\mathrm{P}$ ATIENTS who present with a poor neurological grade after aneurysmal subarachnoid hemorrhage (SAH) often have poor outcomes. Intrasylvian hematoma (ISH) is a subtype of intracranial hematoma caused by aneurysmal rupture and has distinct features.,18,19 Poor-grade SAH with ISH has been considered to be less associated with severe initial injury and regarded as a potential responder to intensive treatment., ${ }^{2,19,20}$ The purpose of this study is to clarify whether the presence of ISH predicts good functional recovery from poor-grade aneurysmal SAH.

\section{Methods}

The study is reported based on criteria from the STROBE (Strengthening the Reporting of Observational

ABBREVIATIONS ICH = intracerebral hematoma; ISH = intrasylvian hematoma; $m R S=$ modified Rankin Scale; SAH = subarachnoid hemorrhage; WFNS = World Federation of Neurosurgical Societies.

SUBMITTED July 17, 2014. ACCEPTED October 21, 2014.

INCLUDE WHEN CITING Published online May 22, 2015; DOI: 10.3171/2014.10.JNS141658.

DISCLOSURE The authors report no conflict of interest concerning the materials or methods used in this study or the findings specified in this paper. 
Study in Epidemiology) statement. ${ }^{23}$ The study protocol was approved by the Kurashiki Central Hospital Research Ethics Committee, and informed consent was obtained from all competent patients or from a relative of patients incapacitated by their SAH.

\section{Patient Selection and Clinical Evaluation}

A prospectively maintained aneurysmal SAH database at our institution comprised 287 patients who were treated within 72 hours after symptom onset between January 2008 and December 2013. Patients were excluded from surgical or endovascular intervention if they had obvious evidence of irreparable brainstem injury, or if next of kin indicated limits on therapeutic interventions. Among them, 97 consecutive patients with initial clinical condition of World Federation of Neurosurgical Societies (WFNS) Grade IV or V were retrospectively reviewed. ${ }^{4}$

Age, sex, hypertension and/or Type 2 diabetes status, preoperative antiplatelet or anticoagulant use, size and location (anterior or posterior circulation) of the aneurysm, treatment modality (clip or coil), intraventricular hemorrhage, and subarachnoid clot burden by semiquantitative Hijdra scale ${ }^{9}$ were analyzed to identify characteristics of SAH with ISH. Subsequently, to assess the contribution of ISH to the clinical course and complications, the following factors were analyzed: initial neurological status according to WFNS grade (Grade IV vs V), decision to undertake external decompression at surgery, presence of initial cerebral edema, procedure-related complications, symptomatic vasospasm, and chronic hydrocephalus. Patients who died before evaluation for symptomatic vasospasm $(n=11)$ and chronic hydrocephalus $(n=14)$ were excluded from these analyses. Procedure-related complications were defined as symptomatic postoperative hematoma formation or cerebral infarction in cases of surgical clipping, vascular perforation, hematoma growth unrelated to aneurysmal rebleeding, ${ }^{5}$ or symptomatic thromboembolic event in cases of endovascular coiling. Symptomatic vasospasm was defined as clinical symptoms, such as confusion or decline in level of consciousness, or focal deficits not clinically or radiographically attributable to other causes, occurring between Days 4 and 14 after SAH.

Functional outcome of the patient at 6 months after discharge was determined using the modified Rankin Scale (mRS) administered by a physician (S.Y.) blinded to the patient's history, and outcome was dichotomized into good (mRS scores of 0-3) and poor (mRS scores of 4-6). The relationship between ISH and recovery to good functional or discharge outcome was analyzed together with the other covariates, including younger age and better initial neurological grade (WFNS Grade IV vs V), which are known predictors of favorable outcomes of patients with poor-grade $\mathrm{SAH} .{ }^{15}$

\section{Radiological Evaluation}

All patients underwent head CT scanning on admission. We observed that an intrasylvian thick clot was most easily identified between the insula and the temporal lobe, and started to show mass effect when its short axis was 10-12 mm long. Therefore, in this study, the presence of ISH was defined as the massive hematoma centering on the sylvian fissure and measuring longer than $15 \mathrm{~mm}$ along the short axis between the insula and the temporal lobe, and determined by an independent neuroradiologist (S.N.) blinded to patients' characteristics and outcomes (Fig. 1). In the non-ISH group, an intracerebral hematoma (ICH) in the brain parenchyma that measured longer than $15 \mathrm{~mm}$ on the short axis was also recorded (Fig. 1B).

All patients also underwent head CT scanning on the day after surgery and at discharge. The volume of the hypodense area on CT imaging was determined by perimeter tracing conducted by an examiner (K.H.) blinded to clinical characteristics. The hypodense area on the head CT scan was manually traced on each individual slice using ViewR software (GE Yokogawa Medical) and was multiplied by slice thickness. The total hypodense area for each patient was calculated as the sum of all individual hypodense volumes..$^{12}$ In the subgroup in which the initial cerebral edema volume was greater than the median volume (18 ml), chronological change of the cerebral edema was analyzed by comparing hypodense areas on the postoperative Day 1 and discharge CT scans.

\section{Treatment Protocol}

During this period, we pursued an early treatment regimen of patients with $\mathrm{SAH}$. Patients were assigned to undergo surgical clipping or endovascular coiling, which was determined according to discussions between surgical and endovascular teams using the initial head CT scan and 3D CT angiogram. When assigned to surgical clipping, patients were immediately transferred to the operation room without undergoing catheter angiography. Patients who were assigned to endovascular coiling or who had no detectable aneurysms on 3D CT angiography were transferred to the angiography suite. The cases requiring hematoma evacuation were preferably assigned to surgical clipping, and external decompression was performed according to brain swelling at operation.

Vasospasm was prevented by a continuous cisternal irrigation protocol with milrinone, which is a bipyridine methyl carbonitrile analog of amrinone and has inotropic and vasodilating action. ${ }^{1,17}$ When angiographic vasospasm was observed on 3D CT angiograms taken every 3 to 4 days, the irrigation solution consisted of lactated Ringer solution, urokinase $(120 \mathrm{IU} / \mathrm{ml})$, ascorbic acid $(3.5 \mathrm{mg} /$ $\mathrm{ml})$, and milrinone $(3.6 \mu \mathrm{g} / \mathrm{ml})$, which was continuously infused between the inlet and outlet tubes inserted in the subarachnoid space until Day 14 after SAH. Intravenous fasudil chloride was also administrated during this period. Percutaneous transluminal angioplasty for vasospasm was not performed in this cohort, because symptoms were mild or because vasospasm occurred in more peripheral vessels.

\section{Data Analysis}

Quantitative variables are expressed as the mean \pm SD or the median value \pm quartile deviation, as appropriate. Univariate logistic regression and chi-square tests were performed to evaluate covariates for binary, categorical, dependent variables, as appropriate. The normality of the data was evaluated using the Shapiro-Wilk test. Nonnormally distributed variables related to the hypodense area 

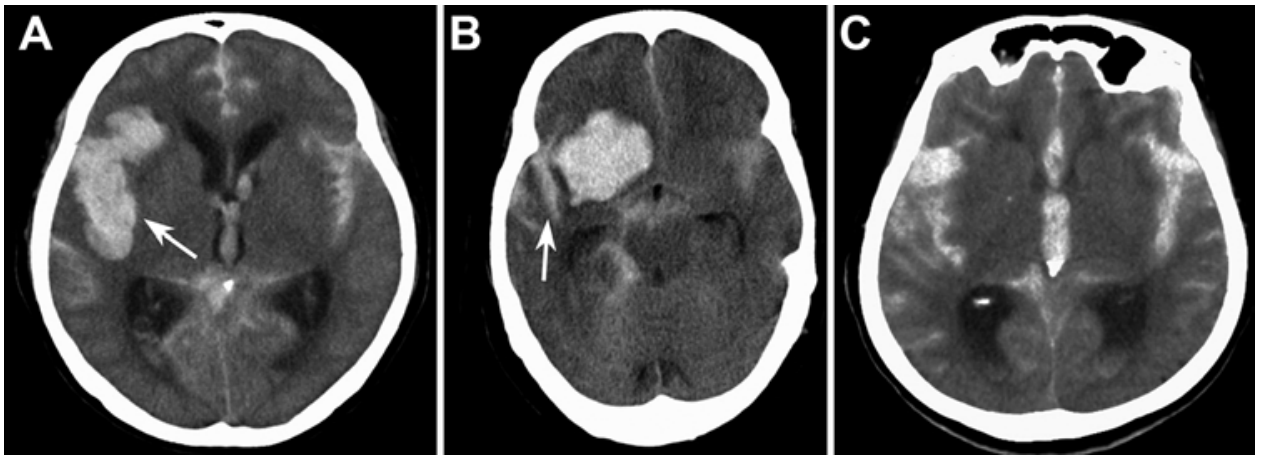

FIG. 1. Head CT scans showing 3 possible patterns of hematoma distribution in poor-grade SAH. A: A dense ISH in which the length of the hematoma's short axis between the temporal lobe and the insula is longer than $15 \mathrm{~mm}$ (arrow). B: A spherical ICH, the location of which is distinct from its associated sylvian fissure subarachnoid clot, which is thin (arrow). C: A diffuse thick subarachnoid clot with intraventricular hemorrhage.

on the head CT scans were compared between patients with and those without ISH using the Mann-Whitney Utest. Significant correlation of ISH with patients' clinical course or good functional outcome was evaluated by using univariate logistic regression. Only variables with $\mathrm{p}<0.20$ in the univariate analysis were included in the multivariate logistic regression model-building process. Models were built using forward/backward stepwise logistic regression, with variables entered into the model and removed at a 0.20 significance level. Values of $\mathrm{p}<0.05$ were considered statistically significant. The OR and $95 \%$ CI were also determined in the logistic regression analysis. Cohen $\kappa$ value was calculated to quantify the level of agreement regarding the presence of ISH between 2 observers (S.N. and K.H.). A value was interpreted as poor $(<0.20)$, fair (0.21-0.40), moderate (0.41-0.60), substantial (0.61-0.80), or almost perfect (0.81-1.0). ${ }^{11}$ Commercially available software (SPSS version 20; IBM Corp.) was used for all statistical analyses.

\section{Results}

\section{ISH and Baseline Characteristics}

Characteristics of patients and incidence of ISH in 97 poor-grade (WFNS Grade IV or V) aneurysmal SAHs are summarized in Table 1. ISH was observed in 14 of 97 cases (14\%) of poor-grade SAH. A representative case of SAH with ISH is shown in Fig. 2. All 14 cases with ISH were caused by ruptured middle cerebral artery aneurysms and treated with surgical clipping without catheter angiography. ICHs longer than $15 \mathrm{~mm}$ along the short axis were observed in 18 of 83 cases (21\%) in the non-ISH group. Temporal lobe ICH was observed in 4 cases, of which 3 were associated with diffuse SAH and 1 was associated with minor SAH.

Our early treatment regimen enabled ultra-early surgery within 6 hours of onset in 47 of 64 cases (73\%) of surgically treated poor-grade SAH and in 13 of 14 cases $(93 \%)$ of SAH with ISH. The baseline characteristics of patients were not significantly different between the ISH and non-ISH groups. Surgical clipping was predominantly performed in the ISH group. The Cohen $\kappa$ value qualifying the level of agreement between observers was 0.78 for presence of ISH on the head CT scan on admission, indicating a substantial degree of agreement.

\section{ISH and Clinical Courses}

Tables 2 and 3 show the results of univariate and multivariate analyses for contribution of ISH to clinical courses and complications, respectively. ISH was not significantly associated with poorer initial neurological grade (WFNS Grade V vs IV), procedure-related complications, symptomatic vasospasm, or chronic hydrocephalus in the univariate logistic regression analysis. Procedure-related complications were observed in 3 of 14 cases $(21 \%)$ in the ISH group and in 21 of 83 cases $(25 \%)$ in the nonISH group (Table 4). Of these cases with complications, 2 $(14 \%)$ in the ISH group and $14(17 \%)$ in the non-ISH group had poor outcomes (mRS scores of 4-6 at 6 months). Symptomatic vasospasm occurred in 2 of 13 cases $(15 \%)$ in the ISH group and in 17 of 73 cases (23\%) in the nonISH group. Symptomatic vasospasm in the ISH group presented with focal neurological worsening, which resolved over time. Global delayed cerebral infarction as a result

TABLE 1. Characteristics of patients with poor-grade SAH with or without ISH*

\begin{tabular}{|c|c|c|c|}
\hline \multirow[b]{2}{*}{ Characteristic } & \multicolumn{2}{|c|}{ SAH Group $(n=97)$} & \multirow[b]{2}{*}{$\mathrm{p}$ Value } \\
\hline & $\mathrm{ISH}(n=14)$ & $\begin{array}{c}\text { Non-ISH } \\
(n=83)\end{array}$ & \\
\hline Age, yrs & $63.4 \pm 12.8$ & $69.1 \pm 13.1$ & 0.13 \\
\hline Female sex & $9(64.3)$ & $60(72.3)$ & 0.54 \\
\hline Hypertension & $7(50.0)$ & $33(40.0)$ & 0.42 \\
\hline Type 2 diabetes & $0(0)$ & $4(5.2)$ & 0.20 \\
\hline Antiplatelet/anticoagulant use & $0(0)$ & $9(10.8)$ & 0.11 \\
\hline Anterior circulation location & $14(100)$ & $71(85.5)$ & 0.13 \\
\hline Surgical clipping & $14(100)$ & $50(60.2)$ & 0.005 \\
\hline IVH & $9(64.3)$ & $60(72.3)$ & 0.54 \\
\hline Mean aneurysm size, $\mathrm{mm}$ & $6.8 \pm 4.9$ & $6.2 \pm 3.0$ & 0.56 \\
\hline Mean Hijdra Scale score & $25.6 \pm 5.2$ & $23.4 \pm 7.6$ & 0.31 \\
\hline
\end{tabular}



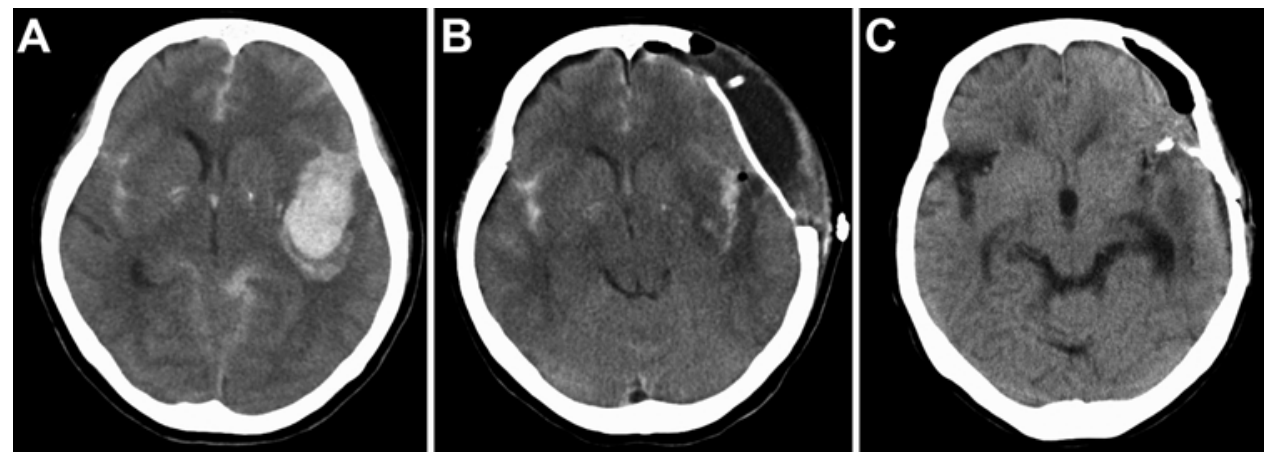

FIG. 2. Head CT scans obtained in a 67-year-old man with WFNS Grade IV SAH, right-sided motor weakness, and complete aphasia. A: Head CT scan on admission demonstrating diffuse SAH with left ISH. B: Head CT scan on the day after surgery, revealing evacuation of hematoma and external decompression. Note the cerebral edema around the sylvian fissure. C: Head CT scan before discharge showing an area of mild hypodensity in the temporal lobe. The patient was discharged to home with mild motor aphasia. His mRS score at discharge was 2.

of diffuse severe vasospasm occurred in 8 patients in the non-ISH group. Of these, 2 patients died of global cerebral ischemia (mRS score of 6), and 6 patients became fully dependent (mRS score of 5).

On the other hand, the presence of ISH was more likely associated with the decision to perform external decompression during surgery (OR 35.5; 95\% CI 7.05-179; $\mathrm{p}<$ 0.001 , univariate logistic regression). SAH patients with ISH were also more likely to have initial cerebral edema ( $\mathrm{p}<0.001$, Mann-Whitney U-test), documented as the hypodensity volumes on the head CT scan obtained the day after surgery. ISH remained an independent contributing factor for the decision to perform external decompression and for initial cerebral edema volume greater than the median volume $(18 \mathrm{ml})$ by multivariable analysis (Table 3). Chronological change of the cerebral edema was analyzed in the subgroup in which the initial cerebral edema volume was greater than the median volume $(18 \mathrm{ml})$. In the ISH group, the hypodense area on CT scans decreased over time by a median of $18 \mathrm{ml}( \pm 7.75 \mathrm{ml}$ [quartile deviation]), whereas in the non-ISH group, this area increased by a median of $17 \mathrm{ml}$ ( $\pm 29.5 \mathrm{ml}$ [quartile deviation]). There was a significant difference between the 2 groups ( $p<0.001$, Mann-Whitney U-test). Initial severe unilateral motor weakness (Grade 0-2/5) improved to function- al strength (Grade 4-5/5) at discharge in 6 of 9 patients $(67 \%)$ in the ISH group.

\section{ISH and Favorable Outcomes}

Functional outcome at 6 months after discharge in the ISH and non-ISH groups is summarized in Fig. 3. Table 5 shows the results of univariate regression analysis of factors for favorable functional outcome (mRS scores of $0-3)$. Favorable functional outcome was observed in 41 patients $(42 \%)$ with poor-grade SAH. Univariate analysis demonstrated that ISH was significantly associated with favorable functional outcome (OR 6.48; 95\% CI 1.67-25.1; $\mathrm{p}=0.007$ ). WFNS Grade IV (vs Grade V; OR 2.93; 95\% CI $1.27-6.79 ; p=0.012)$ and younger age (OR $1.05 ; 95 \%$ CI $1.02-1.09 ; \mathrm{p}=0.004)$ were also significant predictors of favorable outcome. In the multivariable logistic regression analysis, ISH remained an independent predictor of favorable functional outcome with an OR of 5.75 (95\% CI $1.36-24.3 ; p=0.017$ ) after adjustment for potential confounders such as WFNS Grade IV (vs Grade V), younger age, and female sex (Table 6).

\section{Discussion}

In this study, we characterized ISH as independently

TABLE 2. Clinical courses and complications in patients with poor-grade SAH with or without ISH*

\begin{tabular}{|c|c|c|c|c|}
\hline \multirow[b]{2}{*}{ Clinical Courses \& Complications } & \multicolumn{2}{|c|}{ SAH Group $(n=97)$} & \multirow[b]{2}{*}{ OR $(95 \% \mathrm{Cl})$} & \multirow[b]{2}{*}{$p$ Value $†$} \\
\hline & $\mathrm{ISH}(\mathrm{n}=14)$ & Non-ISH $(n=83)$ & & \\
\hline WFNS Grade V & $7(50.0)$ & $50(60.2)$ & $0.66(0.21-2.06) \ddagger$ & 0.47 \\
\hline Decision of external decompression & $12(85.7)$ & $12(14.5)$ & $35.5(7.05-179)$ & $<0.001$ \\
\hline Median cerebral edema vol, $\mathrm{ml}$ & $48.5 \pm 13.5$ & $15 \pm 14$ & & $<0.001 \S$ \\
\hline Cerebral edema vol $>18 \mathrm{ml}$ & $13(92.9)$ & $33(40.0)$ & $17.8(2.23-143)$ & 0.007 \\
\hline Procedure-related complications & $3(21.4)$ & $21(25.3)$ & $0.76(0.19-2.97)$ & 0.69 \\
\hline Symptomatic vasospasm & $2(15.3)$ & $17(23.2)$ & $0.65(0.13-3.17)$ & 0.59 \\
\hline Chronic hydrocephalus & $6(46.2)$ & $27(38.6)$ & $1.56(0.49-4.93)$ & 0.45 \\
\hline
\end{tabular}


TABLE 3. Multivariable analysis of contribution of ISH to clinical courses and complications

\begin{tabular}{lcr}
\hline \multicolumn{1}{c}{ Dependent Variable } & $\begin{array}{c}\text { Adjusted OR } \\
(95 \% \mathrm{Cl})\end{array}$ & p Value* \\
\hline Decision of external decompression† & $35.5(7.05-179)$ & $<0.001$ \\
\hline Initial cerebral edema vol $>18 \mathrm{ml} \dagger$ & $17.8(2.23-143)$ & $\mathbf{0 . 0 0 7}$ \\
\hline
\end{tabular}

* Significant difference by univariate analysis $(p<0.05)$ indicated by boldface.

$\dagger$ Adjusted for age, sex, and presence of intraventricular hemorrhage.

$\ddagger$ Adjusted for age and history of hypertension.

associated with good functional recovery from poor initial neurological grade SAH. Initial cerebral edema significantly improved over time in patients with ISH, which might have contributed to favorable functional outcomes in this group.

Poor-grade SAH is categorized into SAH with ISH, SAH with ICH, and diffuse SAH and/or intraventricular hemorrhage without hematoma (Fig. 1). ${ }^{24}$ In diffuse SAH and/or intraventricular hemorrhage without hematoma, a poor initial neurological grade reflects entire brain hypoperfusion or hypoxia, due to global intracranial pressure surge or cardiorespiratory arrest, respectively, suggesting brain function has been broadly and irreversibly damaged. In SAH with ICH in the brain parenchyma, destruction of substantial brain volume results in a severe, permanent neurological deficit even when local intracranial pressure elevation is properly controlled by decompression. On the the other hand, in SAH with ISH, an initial poor neurological grade may represent states of reversible brain dysfunction secondary to the combined impact of intracranial pressure elevation and localized subpial brain disruption, neither of which alone is necessarily severe enough to lead to permanent neuronal damage of the underlying affected regions. Temporal lobe ICH with minor subarachnoid clot has also been reported to have better outcomes. ${ }^{19}$ However, in our cohort of patients with poor-grade $\mathrm{SAH}$, most of the ICHs involved the frontal lobe or were associated with diffuse SAH. Temporal lobe ICH with a minor subarachnoid clot was observed in only 1 case, thus contributing little to poor functional outcomes in the non-ISH group.

The reversibly disrupted brain in SAH is then exposed to the risk of secondary brain injury. There, a vicious spiral works over time between brain swelling and ischemic infarction, with each exacerbating the other in the limited intracranial space..$^{20}$ It was also described as a "feedforward" cycle of brain injury by Fisher and Ojemann: "Increased intracranial pressure creates a vicious cycle of anoxia $\rightarrow$ swelling $\rightarrow$ more anoxia."6 Indeed, Saito et al. reported that delayed postoperative brain swelling (1 week after surgery) more likely developed in patients with SAH with ISH than in patients with SAH with intraparenchymal ICH. ${ }^{18}$ Given less initial brain damage and associated secondary brain swelling, previous studies have aimed at intervening to treat one of these pathological processes by early hematoma evacuation or external decompression and have obtained reasonable outcomes from poor-grade SAH with ISH. ${ }^{2,19-21}$ Our results also supported validity of early decompression for treatment of SAH with ISH.

In this study, hypodense areas on head CT scans decreased over time in the ISH group, suggesting that a con-
TABLE 4. Procedure-related complications of patients with poor-grade SAH with or without ISH

\begin{tabular}{lcc}
\hline \multirow{2}{*}{ Complications } & \multicolumn{2}{c}{ SAH Group $(\mathrm{n}=97)$} \\
\cline { 2 - 3 } & $\mathrm{ISH}(\mathrm{n}=14)$ & Non-ISH $(\mathrm{n}=83)$ \\
\hline Hematoma formation at clipping & 2 & 1 \\
\hline Cerebral infarction at clipping & 1 & 8 \\
\hline Vascular perforation at coiling & $\mathrm{NA}^{*}$ & 3 \\
\hline $\begin{array}{l}\text { Hematoma growth unrelated to } \\
\text { rebleeding at coiling }\end{array}$ & $\mathrm{NA}^{*}$ & 2 \\
\hline Thromboembolic event at coiling & $\mathrm{NA}^{*}$ & 7 \\
\hline $\begin{array}{l}\text { NA }=\text { not applicable. } \\
*\end{array}$ All cases in the ISH group were treated with surgical clipping.
\end{tabular}

siderable amount of initial brain dysfunction was due to reversible cerebral edema. On the other hand, hypodense areas in the non-ISH group increased in volume, suggesting that initial brain damage was more likely irreversible and that new cerebral infarction occurred as a result of the aforementioned vicious spiral. In addition, two-thirds of SAH patients with ISH experienced functional recovery from initial severe unilateral motor weakness, which contributed to favorable functional outcome. Taken together, initial brain damage in the ISH group was more recoverable and secondary brain injury was avoided under our strategy, both from radiological and functional perspectives.

ISH is formed differently than intraparenchymal-type $\mathrm{ICH}$ in aneurysmal SAH, in which the main component of ISH is subpial extension of the subarachnoid clot., ${ }^{5,22} \mathrm{Be}-$ cause ISH exhibits distinct features, evaluating procedurerelated complications of ISH is meaningful. Removal of an ISH is technically more difficult than that of an ICH, due to involvement of fragile pial vessels. ${ }^{18,24}$ In our cohort of patients with ISH, subpial hematoma expanded after uneventful surgery and required repeated craniotomy in 1 case, which may be a specific complication of the ISH group. Complications related to endovascular coiling exclusively occurred in the non-ISH group. However, the overall complication rate and severity were not significantly different between the 2 groups (Table 4 ).

Another major concern in predicting outcomes of SAH is delayed cerebral infarction caused by vasospasm. In this study, the incidence of symptomatic vasospasm was $15 \%$ in the ISH group and 23\% in the non-ISH group in our cohort of patients with poor-grade SAH. Previous studies have described a correlation between thick subarachnoid clots and a high incidence of vasospasm. ${ }^{13,14}$ However, in our cohort of patients with ISH, the incidence of vasospasm was not higher and vasospasm-related neurological deficits were less severe. In patients with ISH, delayed worsening of ipsilateral vasospasm-related symptoms may not be clinically detected, because it is masked by preexisting neurological deficits associated with cerebral edema accompanying ISH. Furthermore, removal of a massive ISH during surgery may represent a high rate of subarachnoid clot clearance, which has also been reported as an independent predictor of less severe vasospasm. ${ }^{10,16}$

Our study has several methodological limitations. First, because of its observational design, findings should 


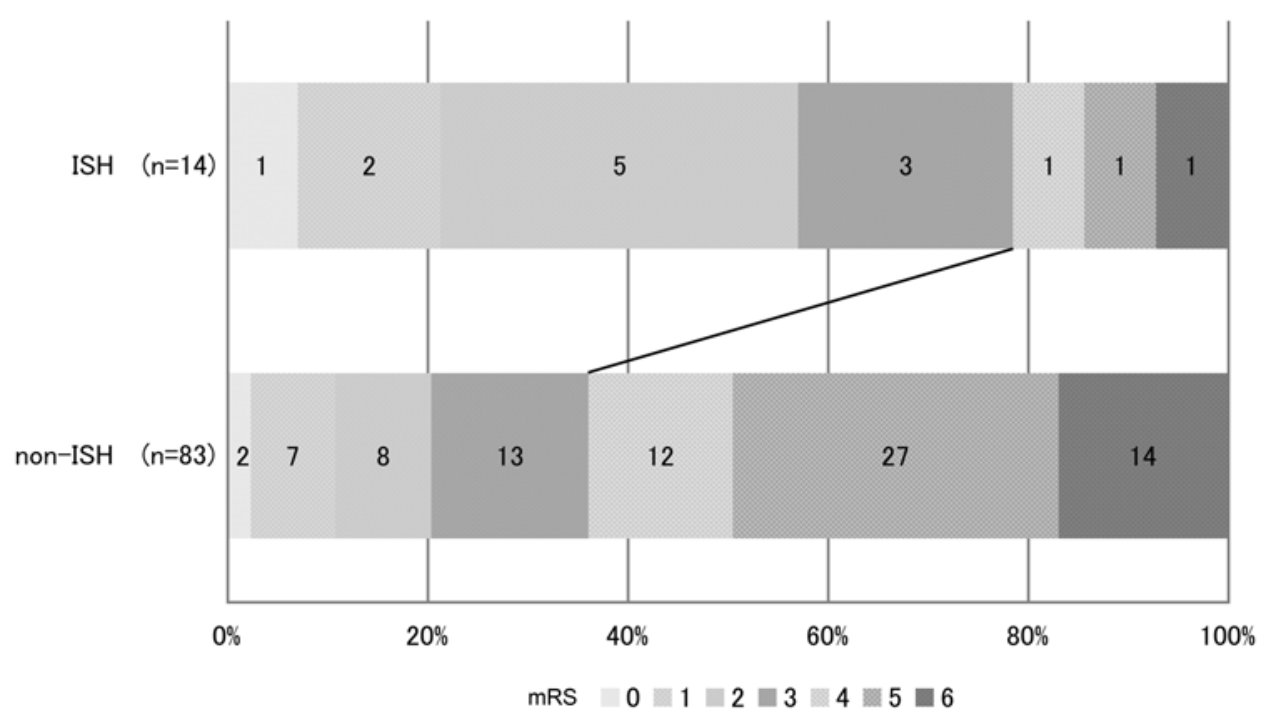

FIG. 3. Graph demonstrating functional outcomes 6 months after discharge in ISH and non-ISH groups. The oblique demarcation line indicates dichotomization between favorable (mRS scores of $0-3$ ) and poor (mRS scores of 4-6) outcomes.

be interpreted cautiously. In a retrospective study, potential interactions among variables may not be completely excluded by multivariable analysis. Second, the patients were treated at a single institution. Third, a consensus on the definition of ISH in SAH is not available in terms of volume, size, and location of the hematoma. Previous studies have defined ISH as having a volume of $10 \mathrm{ml}^{19}$ or 25 $\mathrm{ml},{ }^{20}$ or a maximum diameter of $30 \mathrm{~mm} \cdot{ }^{24}$ An important cutoff point regarding outcomes in spontaneous ICH has been reported as a volume of $25-30 \mathrm{ml} .^{3,7,8}$ However, it is unclear whether this volume can be applied to SAH with ISH. Unlike spontaneous ICH, ISH is often associated with a diffuse subarachnoid clot that modifies intracranial pressure and cerebral circulation. In addition, it is intrinsically difficult to distinguish a subpial hematoma from a subarachnoid clot in ISH. For instance, the presence of ISH may be overestimated in patients with a wide sylvian fissure due to brain atrophy, even though its accompanying subpial hematoma, which is a true component of ISH, is small. Furthermore, the complicated shape of the sylvian fissure disrupts precise measurement of ISH volume, and radiological artifact from skull-base bones on CT imaging is unavoidable when the basal sylvian fissure is evaluated. In this study, we included poor initial neurological grade SAH patients with ISH measuring at least $15 \mathrm{~mm}$ between the temporal lobe and insula. If required, we will continue to refine this inclusion criterion in future studies. Further investigation would be warranted to confirm functional recovery of SAH with ISH and identify causative mechanisms.

\section{Conclusions}

ISH caused by ruptured middle cerebral artery aneu-

TABLE 5. Univariate analysis of factors for favorable functional outcome (mRS scores 0-3 at 6 months)*

\begin{tabular}{|c|c|c|c|c|}
\hline \multirow[b]{2}{*}{ Variable } & \multicolumn{2}{|c|}{ Functional Outcome } & \multirow[b]{2}{*}{ OR $(95 \% \mathrm{Cl})$} & \multirow[b]{2}{*}{$\mathrm{p}$ Valuet: } \\
\hline & Favorable $(n=41)$ & Poor $(n=56)$ & & \\
\hline ISH & $11(26.8)$ & $3(5.4)$ & $6.48(1.67-25.1)$ & 0.007 \\
\hline WFNS Grade IV (vs V) & $23(56.1)$ & $17(30.4)$ & $2.93(1.27-6.79)$ & 0.012 \\
\hline Younger age, yrs & $63.6 \pm 12.8$ & $68.3 \pm 13.1$ & $1.05(1.02-1.09)$ & 0.004 \\
\hline Female sex & $26(63.4)$ & $43(76.8)$ & $0.52(0.22-1.27)$ & 0.15 \\
\hline Hypertension & $15(36.6)$ & $24(42.9)$ & $0.77(0.34-1.76)$ & 0.53 \\
\hline Type 2 diabetes & $1(2.4)$ & $3(5.4)$ & $0.44(0.04-4.41)$ & 0.49 \\
\hline Antiplatelet/anticoagulant use & $3(7.3)$ & $6(10.7)$ & $0.66(0.16-2.80)$ & 0.57 \\
\hline Anterior circulation & $38(92.7)$ & $47(83.9)$ & $2.43(0.61-9.62)$ & 0.21 \\
\hline Surgical clipping & $30(73.2)$ & $35(62.5)$ & $1.64(0.68-3.94)$ & 0.27 \\
\hline IVH & $27(65.9)$ & $42(75.0)$ & $0.64(0.27-1.58)$ & 0.33 \\
\hline Aneurysm size, $\mathrm{mm}$ & $5.9 \pm 3.8$ & $6.6 \pm 2.9$ & $0.94(0.82-1.07)$ & 0.33 \\
\hline Hijdra Scale score & $23.1 \pm 7.0$ & $24.2 \pm 7.6$ & $0.98(0.93-1.04)$ & 0.49 \\
\hline
\end{tabular}

* Data are presented as mean \pm SD or as number $(\%)$ of patients.

$\dagger$ Significant difference by univariate analysis $(p<0.05)$ indicated by boldface.

$\ddagger$ Marginally significant variable $(p<0.20)$ indicated in italics. 
TABLE 6. Multivariable logistic regression analysis of factors for favorable functional outcome

\begin{tabular}{lcc}
\hline \multicolumn{1}{c}{ Variable } & Adjusted OR $(95 \% \mathrm{Cl})$ & p Value* \\
\hline ISH & $5.75(1.36-24.3)$ & $\mathbf{0 . 0 1 7}$ \\
\hline WFNS Grade IV (vs V) & $3.26(1.29-8.24)$ & $\mathbf{0 . 0 1 3}$ \\
\hline Younger age & $1.05(1.01-1.09)$ & $\mathbf{0 . 0 0 7}$ \\
\hline
\end{tabular}

* Significant difference by univariate analysis $(p<0.05)$ indicated by boldface.

rysms predicted good functional recovery from poor-grade $\mathrm{SAH}$. Aggressive treatment is recommended to maximize potential functional recovery of this subgroup of poorgrade SAHs.

\section{Acknowledgment}

We thank Hiroyuki Yamamoto for radiological evaluation and data collection.

\section{References}

1. Arakawa Y, Kikuta K, Hojo M, Goto Y, Yamagata S, Nozaki $\mathrm{K}$, et al: Milrinone reduces cerebral vasospasm after subarachnoid hemorrhage of WFNS grade IV or V. Neurol Med Chir (Tokyo) 44:393-401, 2004

2. Başkaya MK, Menendez JA, Yüceer N, Polin RS, Nanda A: Results of surgical treatment of intrasylvian hematomas due to ruptured intracranial aneurysms. Clin Neurol Neurosurg 103:23-28, 2001

3. Broderick JP, Brott TG, Duldner JE, Tomsick T, Huster G: Volume of intracerebral hemorrhage. A powerful and easyto-use predictor of 30-day mortality. Stroke 24:987-993, 1993

4. Drake CG: Report of World Federation of Neurological Surgeons Committee on a universal subarachnoid hemorrhage grading scale. J Neurosurg 68:985-986, 1988 (Letter)

5. Egashira Y, Yoshimura S, Enomoto Y, Ishiguro M, Asano T, Iwama T: Ultra-early endovascular embolization of ruptured cerebral aneurysm and the increased risk of hematoma growth unrelated to aneurysmal rebleeding. J Neurosurg 118:1003-1008, 2013

6. Fisher CM, Ojemann RG: Bilateral decompressive craniectomy for worsening coma in acute subarachnoid hemorrhage. Observations in support of the procedure. Surg Neurol 41:65-74, 1994

7. Hårdemark HG, Wesslén N, Persson L: Influence of clinical factors, CT findings and early management on outcome in supratentorial intracerebral hemorrhage. Cerebrovasc Dis 9:10-21, 1999

8. Helweg-Larsen S, Sommer W, Strange P, Lester J, Boysen G: Prognosis for patients treated conservatively for spontaneous intracerebral hematomas. Stroke 15:1045-1048, 1984

9. Hijdra A, Brouwers PJ, Vermeulen M, van Gijn J: Grading the amount of blood on computed tomograms after subarachnoid hemorrhage. Stroke 21:1156-1161, 1990

10. Hosoda K, Fujita S, Kawaguchi T, Shose Y, Hamano S, Iwakura M: Effect of clot removal and surgical manipulation on regional cerebral blood flow and delayed vasospasm in early aneurysm surgery for subarachnoid hemorrhage. Surg Neurol 51:81-88, 1999

11. Ibrahim GM, Weidauer S, Macdonald RL: Interobserver variability in the interpretation of computed tomography following aneurysmal subarachnoid hemorrhage. J Neurosurg 115:1191-1196, 2011

12. Kumar A, Brown R, Dhar R, Sampson T, Derdeyn CP, Moran $\mathrm{CJ}$, et al: Early vs. delayed cerebral infarction after aneurysm repair after subarachnoid hemorrhage. Neurosurgery 73:617-623, 2013
13. Macdonald RL: Management of cerebral vasospasm. Neurosurg Rev 29:179-193, 2006

14. Macdonald RL, Rosengart A, Huo D, Karrison T: Factors associated with the development of vasospasm after planned surgical treatment of aneurysmal subarachnoid hemorrhage. J Neurosurg 99:644-652, 2003

15. Mocco J, Ransom ER, Komotar RJ, Schmidt JM, Sciacca RR, Mayer SA, et al: Preoperative prediction of long-term outcome in poor-grade aneurysmal subarachnoid hemorrhage. Neurosurgery 59:529-538, 2006

16. Reilly C, Amidei C, Tolentino J, Jahromi BS, Macdonald RL: Clot volume and clearance rate as independent predictors of vasospasm after aneurysmal subarachnoid hemorrhage. J Neurosurg 101:255-261, 2004

17. Sadamasa N, Yoshida K, Narumi O, Chin M, Yamagata S: Milrinone via lumbar subarachnoid catheter for vasospasm after aneurysmal subarachnoid hemorrhage. Neurocrit Care 21:470-475, 2014

18. Saito A, Akamatsu Y, Mikawa S, Sugawara T, Seki H: Comparison of large intrasylvian and subpial hematomas caused by rupture of middle cerebral artery aneurysm. Neurol Med Chir (Tokyo) 50:281-285, 2010

19. Shimoda M, Oda S, Mamata Y, Tsugane R, Sato O: Surgical indications in patients with an intracerebral hemorrhage due to ruptured middle cerebral artery aneurysm. J Neurosurg 87:170-175, 1997

20. Smith ER, Carter BS, Ogilvy CS: Proposed use of prophylactic decompressive craniectomy in poor-grade aneurysmal subarachnoid hemorrhage patients presenting with associated large sylvian hematomas. Neurosurgery 51:117-124, 2002

21. Su CC, Saito K, Nakagawa A, Endo T, Suzuki Y, Shirane R: Clinical outcome following ultra-early operation for patients with intracerebral hematoma from aneurysm rupture-focussing on the massive intra-sylvian type of subarachnoid hemorrhage. Acta Neurochir Suppl 82:65-69, 2002

22. van Asch CJ, Oudendijk JF, Rinkel GJ, Klijn CJ: Early intracerebral hematoma expansion after aneurysmal rupture. Stroke 41:2592-2595, 2010

23. von Elm E, Altman DG, Egger M, Pocock SJ, Gøtzsche PC, Vandenbroucke JP: The Strengthening the Reporting of Observational Studies in Epidemiology (STROBE) statement: guidelines for reporting observational studies. Lancet 370:1453-1457, 2007

24. Yoshimoto Y, Wakai S, Satoh A, Hirose Y: Intraparenchymal and intrasylvian haematomas secondary to ruptured middle cerebral artery aneurysms: prognostic factors and therapeutic considerations. Br J Neurosurg 13:18-24, 1999

\section{Author Contributions}

Conception and design: Fukuda. Acquisition of data: Fukuda, Hayashi, Moriya, Nakashita. Analysis and interpretation of data: Fukuda, Lo. Drafting the article: Fukuda, Lo. Critically revising the article: all authors. Reviewed submitted version of manuscript: all authors. Approved the final version of the manuscript on behalf of all authors: Fukuda. Statistical analysis: Fukuda, Lo. Administrative/technical/material support: Hayashi, Moriya, Nakashita. Study supervision: Yamagata.

\section{Supplemental Information \\ Current Affiliation}

Dr. Lo: Montreal Neurological Institute and Hospital, McGill University, Montreal, Quebec, Canada.

\section{Correspondence}

Hitoshi Fukuda, Department of Neurosurgery, Kurashiki Central Hospital, 1-1-1, Miwa, Kurashiki City, Okayama 710-0052, Japan.email: fukudaharpseal@gmail.com. 